\title{
Lee classes for pseudocomplemented semilattices, revisited
}

\section{SPINKS AND J. SCHMID}

\begin{abstract}
We give syntactic and semantic characterisations of the Lee classes of the varieties of pseudocomplemented semilattices and pseudocomplemented distributive lattices. The main result clarifies earlier work of K. B. Lee and of the second author.
\end{abstract}

\section{Introduction}

A pseudocomplemented semilattice (PCSL) is an algebra $\left\langle A ; \wedge,{ }^{*}, 0\right\rangle$ where $\langle A ; \wedge, 0\rangle$ is a meet semilattice with zero, and for all $a, b \in A, a \wedge b=0$ iff $a \leq b^{*}$. A pseudocomplemented distributive lattice $(\mathrm{PCDL})$ is an algebra $\left\langle A ; \wedge, \vee,{ }^{*}, 0\right\rangle$ where $\langle A ; \wedge, \vee\rangle$ is a distributive lattice and $\left\langle A ; \wedge,{ }^{*}, 0\right\rangle$ is a pseudocomplemented semilattice. By Balbes and Horn [2, Theorem 1.1] the class $\mathcal{P C S} \mathcal{L}$ (respectively, $\mathcal{P C D} \mathcal{L}$ ) of all PCSLs (respectively, of all PCDLs) is equationally definable.

For each $n \in \omega$, let $\mathbf{B}_{n}$ denote the Boolean lattice with $n$ atoms ( $\mathbf{B}_{0}$ is trivial) and greatest element $e$, and let $\hat{\mathbf{B}}_{n}$ denote the lattice obtained from $\mathbf{B}_{n}$ by adjoining a new greatest element $1 \notin B_{n}$. It is well known that the algebras $\hat{\mathbf{B}}_{n}$ may be viewed either as PCSLs or as PCDLs. Considered as PCSLs (respectively, PCDLs), the algebras $\hat{\mathbf{B}}_{n}$ comprise, to within isomorphism, precisely the finite subdirectly irreducible members of $\mathcal{P C S} \mathcal{L}$ (respectively, $\mathcal{P C D} \mathcal{L}$ ); see Sankappanavar [8, Theorem 1] for the case of PCSLs and Lakser [7, Theorem 2] for the case of PCDLs. The quasivariety $\mathcal{B}_{n}:=\operatorname{ISP}\left(\hat{\mathbf{B}}_{n}\right)$, where $\hat{\mathbf{B}}_{n}$ is understood either as a PCSL or as a PCDL, is known as the $n$-th Lee class.

Lee classes (of PCDLs) were first considered by K. B. Lee in [5]. There, it was shown that each quasivariety $\mathcal{B}_{n}$ of PCDLs is actually a variety. The varieties $\mathcal{B}_{n}$ exhaust the non-trivial proper equational subclasses of $\mathcal{P C D} \mathcal{L}$; thus the lattice of subvarieties of $\mathcal{P C D} \mathcal{L}$ is a chain of order type $\omega+1$. It was further shown in [5] that, for $n \geq 1, \mathcal{B}_{n}$ is axiomatised relative to $\mathcal{P C D} \mathcal{L}$ by a

Presented by J. Raftery.

Received July 7, 2009; accepted in final form December 14, 2009.

2010 Mathematics Subject Classification: Primary: 06A12; Secondary: 06D15.

Key words and phrases: pseudocomplemented semilattice, pseudocomplemented distributive lattice, Lee class.

This paper was written while the first author was a Postdoctoral Research Fellow of the Mathematical Institute, University of Bern. The facilities and assistance provided by the University and by the Institute are gratefully acknowledged. 
single identity in $n$ variables, viz.:

$$
\left(\boldsymbol{x}_{\boldsymbol{1}} \wedge \cdots \wedge \boldsymbol{x}_{\boldsymbol{n}}\right)^{*} \vee\left(\boldsymbol{x}_{\mathbf{1}}{ }^{*} \wedge \cdots \wedge \boldsymbol{x}_{\boldsymbol{n}}\right)^{*} \vee \cdots \vee\left(\boldsymbol{x}_{\mathbf{1}} \wedge \cdots \wedge \boldsymbol{x}_{\boldsymbol{n}}{ }^{*}\right)^{*} \approx \mathbf{0}^{*} . \quad\left(\mathrm{Lee}_{n}\right)
$$

The identity $\left(\right.$ Lee $\left._{n}\right)$ is known as the $n$-th Lee identity.

In contrast, G. T. Jones showed in $[6]$ that $\hat{\mathbf{B}}_{1}$ (considered as a PCSL) generates $\mathcal{P C S} \mathcal{L}$ as a variety. Thus the lattice of subvarieties of $\mathcal{P C S} \mathcal{L}$ is the 3 -chain, and the only non-trivial proper subvariety of $\mathcal{P C S} \mathcal{L}$ is (up to term equivalence) the variety of Boolean algebras. It follows that the quasivarieties $\mathcal{B}_{n}$ (considered as classes of PCSLs) are not equationally definable in general.

Lee classes of PCSLs were investigated by the second author in [9]. There, it was shown that for $n \geq 1$, each Lee identity $\left(\operatorname{Lee}_{n}\right)$ may be reformulated (up to equivalence over $\mathcal{P C D} \mathcal{L}$ ) as a quasi-identity $\left(\lambda_{n}^{\prime}\right)$ in $n+2$ variables over the language $\left\{\wedge,{ }^{*}, \mathbf{0}\right\}$. Notwithstanding these remarks, the $n$-th Lee class (considered as a quasivariety of PCSLs) is not axiomatised relative to $\mathcal{P C S} \mathcal{L}$ by the quasi-identity $\left(\lambda_{n}^{\prime}\right)$. Rather, each PCSL-quasivariety $\mathcal{B}_{n}$ is axiomatised relative to $\mathcal{P C S} \mathcal{L}$ by a single quasi-identity $\left(\mu_{n}\right)$ in $n+4$ variables. The relationship between the quasivarieties $\left\{\mathbf{A} \in \mathcal{P C} \mathcal{S} \mathcal{L}: \mathbf{A} \models\left(\lambda_{n}^{\prime}\right)\right\}$ and $\mathcal{B}_{n+1}$ is described in [10, Proposition 4.1].

The quasi-identities $\left(\mu_{n}\right)$ for $n \geq 1$, given in [9] are slightly technical in nature. Moreover, their meaning is somewhat recondite. The aim of this paper is to show that each Lee class $\mathcal{B}_{n}$ (where $\mathcal{B}_{n}$ is considered either as a quasivariety of PCSLs or as a variety of PCDLs) may be axiomatised relative to $\mathcal{P C S} \mathcal{L}$ or to $\mathcal{P C D} \mathcal{L}$ by a single quasi-identity $\left(\mathrm{L}_{n}\right)$ that, syntactically and semantically, has a simple description.

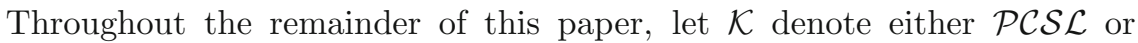
$\mathcal{P C D} \mathcal{L}$, and assume $\mathcal{B}_{n} \subseteq \mathcal{K}$ for each $n \in \omega$. Let $\boldsymbol{x} \backslash \boldsymbol{y}$ abbreviate the $\left\{\wedge,{ }^{*}, \mathbf{0}\right\}$ term $\boldsymbol{x} \wedge \boldsymbol{y}^{*}$. It is easy to see that the term $\boldsymbol{x} \backslash \boldsymbol{y}$ realises the binary $\mathbf{0}^{\mathbf{A}}$ discriminator in the sense of [3] on any bounded chain $\mathbf{A}$ (viewed as a member of $\mathcal{K})$. Consider the family of $\left\{\wedge,{ }^{*}, \mathbf{0}\right\}$-quasi-identities in $n+3$ variables $(0<n<\omega)$ :

$$
\begin{gathered}
\boldsymbol{x} \backslash \boldsymbol{y} \approx \boldsymbol{y} \backslash \boldsymbol{x} \Longrightarrow \boldsymbol{x} \approx \boldsymbol{y}, \\
\&_{i=0}^{n} \boldsymbol{x} \backslash \boldsymbol{z}_{\boldsymbol{i}} \approx \boldsymbol{y} \backslash \boldsymbol{z}_{\boldsymbol{i}} \quad \& \quad \underset{0 \leq j<k \leq n}{\&_{j} \wedge z_{k} \approx \mathbf{0}} \Longrightarrow \boldsymbol{x} \approx \boldsymbol{y} .
\end{gathered}
$$

For any $\mathbf{A} \in \mathcal{K}$, it is clear that $\mathbf{A} \in \mathcal{B}_{0}$ iff $\mathbf{A} \models\left(\mathrm{L}_{0}\right)$ iff for all $a, b \in A$, $\Theta^{\mathbf{A}}(0, a)=\Theta^{\mathbf{A}}(0, b)$ implies $a=b$ (apply [1, Theorem 2.1]).

The main result of this paper asserts

Theorem 1.1. Let $\mathbf{A} \in \mathcal{K}$. Then the following are equivalent for $0<n<\omega$ :

(1) $\mathbf{A} \in \mathcal{B}_{n}$.

(2) $\mathbf{A} \models\left(\mu_{n}\right)$.

(3) $\mathbf{A} \models\left(\mathrm{L}_{n}\right)$. 
(4) For any family of pairwise disjoint elements $\left\{z_{0}, \ldots, z_{n}\right\} \subseteq A$,

$$
\bigcap_{i=0}^{n}\left\{\Theta^{\mathbf{A}}\left(0, z_{i}\right)\right\}=\Delta_{A} .
$$

If $\mathbf{A} \in \mathcal{P C D} \mathcal{L}$, then conditions (1)-(4) are equivalent to

(5) $\mathbf{A} \models\left(\lambda_{n}^{\prime}\right)$.

(6) $\mathbf{A} \models\left(\mathrm{Lee}_{n}\right)$.

The remainder of this paper is devoted to proving Theorem 1.1.

\section{Proof of the Main Theorem}

For $\mathbf{A} \in \mathcal{P C S} \mathcal{L}$, the equivalence $(1) \Leftrightarrow(2)$ is $[9$, Theorem B]. For $\mathbf{A} \in$ $\mathcal{P C D} \mathcal{L}$, the equivalence $(2) \Leftrightarrow(5)$ is [9, Corollary 4.2], while the equivalence $(5) \Leftrightarrow(6)$ is observed in $[9$, Section 2, p. 225]. Of course, the equivalence (1) $\Leftrightarrow(6)$ for $\mathbf{A} \in \mathcal{P C D} \mathcal{L}$ is well known. Thus it remains only to establish the equivalences $(1) \Leftrightarrow(3)$ and $(3) \Leftrightarrow(4)$ for $\mathbf{A} \in \mathcal{K}$.

Apropos the next lemma, recall that $\mathcal{K}$ satisfies the identities:

$$
\begin{gathered}
\mathbf{0}^{* *} \approx \mathbf{0}, \\
\boldsymbol{x}^{* * *} \approx \boldsymbol{x}, \\
\boldsymbol{x} \wedge \mathbf{0}^{*} \approx \boldsymbol{x}^{*}, \\
(\boldsymbol{x} \wedge \boldsymbol{y})^{* *} \approx \boldsymbol{x}^{* *} \wedge \boldsymbol{y}^{* *}, \\
\boldsymbol{x}^{*} \wedge \boldsymbol{y}^{*} \approx\left(\boldsymbol{x} \wedge \boldsymbol{y}^{*}\right)^{*} \wedge \boldsymbol{y}^{*} .
\end{gathered}
$$

Recall further that for any $\mathbf{A} \in \mathcal{K}$, the skeleton of $\mathbf{A}$ is $\mathbf{S}(\mathbf{A}):=\left\{a^{*}: a \in A\right\}$. Of course, $a \in \mathrm{S}(\mathbf{A})$ iff $a=a^{* *}$.

Lemma 2.1. Let $\mathbf{A} \in \mathcal{K}$ and let $0<n<\omega$. Then $\mathbf{A} \models\left(\mathrm{L}_{n}\right)$ iff for all $x, y \in A$ and $z_{0}, \ldots, z_{n} \in \mathrm{S}(\mathbf{A})$,

$$
\begin{aligned}
& x \backslash z_{i}=y \backslash z_{i} \text { for all } i=0, \ldots, n \\
& \quad \text { and } z_{j} \wedge z_{k}=0 \text { for all } 0 \leq j<k \leq n \text { implies } x=y .
\end{aligned}
$$

Proof. $\mathbf{A} \models\left(\mathrm{L}_{n}\right)$ implies $\mathbf{A}$ satisfies $\left(\mathrm{L}_{n}^{\prime}\right)$ by specialisation. Conversely, suppose $\mathbf{A}$ satisfies the implication $\left(\mathrm{L}_{n}^{\prime}\right)$. Assume $x, y \in A$ and $z_{0}, \ldots, z_{n} \in A$ are such that $x \backslash z_{i}=y \backslash z_{i}$ for all $i=0, \ldots, n$ and $z_{j} \wedge z_{k}=0$ for all $0 \leq j<k \leq n$. Notice now that for all $i=0, \ldots, n,\left(z_{i}^{* *}\right) \stackrel{* *}{=} z_{i}^{* *} ;$ thus $z_{0}^{* *}, \ldots, z_{n}^{* *} \in \mathrm{S}(\mathbf{A})$. Moreover, observe that

$$
x \backslash z_{i}^{* *}=x \wedge z_{i}^{* * *} \stackrel{(2.2)}{=} x \wedge z_{i}^{*} \stackrel{(\text { Hyp. })}{=} y \wedge z_{i}^{*} \stackrel{(2.2)}{=} y \wedge z_{i}^{* * *}=y \backslash z_{i}^{* *}
$$

for all $i=0, \ldots, n$, and further, that

$$
0 \stackrel{(2.1)}{=} 0^{* *} \stackrel{(\mathrm{Hyp} .)}{=}\left(z_{j} \wedge z_{k}\right) \stackrel{* *(2.4)}{=} z_{j}^{* *} \wedge z_{k}^{* *}, \quad \text { for all } 0 \leq j<k \leq n .
$$

By $\left(\mathrm{L}_{n}^{\prime}\right)$, we conclude that $x=y$. Thus $\mathbf{A} \models\left(\mathrm{L}_{n}\right)$, completing the proof. 
The well known Glivenko-Frink theorem asserts (in part) that, for any algebra $\mathbf{A} \in \mathcal{K}$, the underlying semilattice ordering of $\mathbf{A}$ partially orders $\mathrm{S}(\mathbf{A})$, and makes $\mathrm{S}(\mathbf{A})$ into a Boolean lattice (which is denoted here by $\mathbf{S}(\mathbf{A})$ ).

The proof of the next lemma is inspired by [4, Lemma 15.3].

Lemma 2.2. $\hat{\mathbf{B}}_{r} \models\left(\mathrm{L}_{n}\right)$ for any $0 \leq r \leq n$, where $1 \leq n \in \omega$.

Proof. The result is clearly true when $r=0$, so we may assume $r \geq 1$. Assume $x, y \in \hat{B}_{r}$ and $z_{0}, \ldots, z_{n} \in \mathrm{S}\left(\hat{\mathbf{B}}_{r}\right)$ are such that $x \backslash z_{i}=y \backslash z_{i}$ for all $i=0, \ldots, n$ and $z_{j} \wedge z_{k}=0$ for all $0 \leq j<k \leq n$. Note now that $\mathbf{S}\left(\hat{\mathbf{B}}_{r}\right) \cong \mathbf{B}_{r}$, where $\mathbf{B}_{r}$ is the Boolean lattice with $r$ atoms. Since $\mathbf{B}_{r}$ does not have $n+1$ pairwise disjoint non-zero elements (as $r \leq n$ ), we conclude there is a $z_{l}, l \in\{0, \ldots, n\}$, such that $z_{l}=0$. But then

$$
x \stackrel{(2.3)}{=} x \wedge 0^{*}=x \wedge z_{l}^{*}=x \backslash z_{l} \stackrel{(\text { Hyp. })}{=} y \backslash z_{l}=y \wedge z_{l}^{*}=y \wedge 0^{*} \stackrel{(2.3)}{=} y .
$$

Thus the implication $\left(\mathrm{L}_{n}^{\prime}\right)$ holds in $\hat{\mathbf{B}}_{r}$. By Lemma 2.1, we conclude that $\hat{\mathbf{B}}_{r} \models\left(\mathrm{L}_{n}\right)$.

Lemma 2.3. For any $1 \leq n \in \omega, \hat{\mathbf{B}}_{n+1} \not \forall\left(\mathrm{L}_{n}\right)$. Hence $\hat{\mathbf{B}}_{r} \forall\left(\mathrm{L}_{n}\right)$ for any $r>n$.

Proof. Let $z_{0}, \ldots, z_{n} \in B_{n+1}$ be the $n+1$ atoms of the Boolean lattice $\mathbf{B}_{n+1}$. Then certainly $z_{j} \wedge z_{k}=0$ for all $0 \leq j<k \leq n$. Since each $z_{i} \neq 0$ for $i \in\{0, \ldots, n\}$, each $z_{i}^{*}<e$, whence $e \backslash z_{i}=z_{i}^{*}=1 \backslash z_{i}$ for all $i=0, \ldots, n$. Thus the premisses of $\left(\mathrm{L}_{n}\right)$ are satisfied in $\hat{\mathbf{B}}_{n+1}$. However, the conclusion of $\left(\mathrm{L}_{n}\right)$ fails to hold in $\hat{\mathbf{B}}_{n+1}$ since $e \neq 1$. Thus $\hat{\mathbf{B}}_{n+1} \not \models\left(\mathrm{L}_{n}\right)$. The final statement now follows since $\hat{\mathbf{B}}_{j}$ lies canonically embedded in $\hat{\mathbf{B}}_{j+1}$ for each $j \in \omega$.

The next lemma is proved in the context of PCSLs in [9, Lemma 4.1], but the proof given there extends effortlessly to PCDLs, because (by [5, Lemma 2]) $\mathcal{P C D} \mathcal{L}$ is locally finite.

Lemma 2.4. Let $\mathbf{A} \in \mathcal{K}$ and let $n \in \omega$. Then $\mathbf{A} \in \mathcal{B}_{n}$ iff $\mathbf{A}_{\text {fin }} \in \mathcal{B}_{n}$ for every finite subalgebra $\mathbf{A}_{\text {fin }} \leq \mathbf{A}$.

Proposition 2.5. For any $n \in \omega, \mathbf{A} \in \mathcal{B}_{n}$ iff $\mathbf{A} \models\left(\mathrm{L}_{n}\right)$.

Proof. The case $n=0$ has been observed previously, so we may assume $n \geq 1$.

By Lemma 2.2, $\hat{\mathbf{B}}_{n} \models\left(\mathrm{L}_{n}\right)$. But then $\mathcal{B}_{n} \models\left(\mathrm{L}_{n}\right)$ as quasi-identities are preserved under $\mathbf{I}, \mathbf{S}$, and $\mathbf{P}$. Hence $\mathbf{A} \in \mathcal{B}_{n}$ implies $\mathbf{A} \models\left(\mathrm{L}_{n}\right)$.

Conversely, suppose $\mathbf{A} \in \mathcal{K}$ is such that $\mathbf{A} \notin \mathcal{B}_{n}$. We must show $\mathbf{A} \not \models\left(\mathrm{L}_{n}\right)$. By Lemma 2.4, there is a finite subalgebra $\mathbf{C} \leq \mathbf{A}$ such that $\mathbf{C} \notin \mathcal{B}_{n}$. Let $\mathbf{C} \leq \mathbf{C}_{1} \times \cdots \times \mathbf{C}_{k}$ be an irredundant subdirect representation of $\mathbf{C}$ with subdirectly irreducible factors. Since $\mathbf{C} \notin \mathcal{B}_{n}$, there must be a factor $\mathbf{C}_{i}$ for $i \in\{1, \ldots, k\}$, such that $\mathbf{C}_{i} \cong \hat{\mathbf{B}}_{r}$ with $r>n$. By Lemma 2.3, $\mathbf{C}_{i} \not \models\left(\mathrm{L}_{n}\right)$. This implies $\mathbf{C} \not \forall\left(\mathrm{L}_{n}\right)$, whence $\mathbf{A} \not \forall\left(\mathrm{L}_{n}\right)$ as $\mathbf{C} \leq \mathbf{A}$.

Proposition 2.5 establishes the equivalence $(1) \Leftrightarrow(3)$ in Theorem 1.1 . 
Observe next that $\mathcal{K}$ satisfies the identity

$$
\boldsymbol{f}\left(\boldsymbol{x}_{1}, \ldots, \boldsymbol{x}_{\boldsymbol{n}}\right) \backslash \boldsymbol{y} \approx \boldsymbol{f}\left(\boldsymbol{x}_{\boldsymbol{1}} \backslash \boldsymbol{y}, \ldots, \boldsymbol{x}_{\boldsymbol{n}} \backslash \boldsymbol{y}\right) \backslash \boldsymbol{y}
$$

for every $n$-ary operation symbol $\boldsymbol{f}$ in the type of $\mathcal{K} ; c f$. [3, Theorem 5.1]. This readily yields

Lemma 2.6. For any $\mathbf{A} \in \mathcal{K}$ and fixed $c \in A$, the relation $\theta_{c}$, defined for all $a, b \in A$ by $a \equiv b\left(\bmod \theta_{c}\right)$ iff $a \backslash c=b \backslash c$, is a congruence on $\mathbf{A}$. Moreover, $\theta_{c}=\Theta^{\mathbf{A}}(0, c)$.

Proposition 2.7. Let $\mathbf{A} \in \mathcal{K}$. Then $\mathbf{A} \models\left(\mathrm{L}_{n}\right)$ iff for any family of pairwise disjoint elements $\left\{z_{0}, \ldots, z_{n}\right\} \subseteq A$, we have $\bigcap_{i=0}^{n}\left\{\Theta^{\mathbf{A}}\left(0, z_{i}\right)\right\}=\Delta_{A}$.

Proof. Suppose $\mathbf{A} \models\left(\mathrm{L}_{n}\right)$. Let $\left\{z_{0}, \ldots, z_{n}\right\} \subseteq A$ be any family of pairwise disjoint elements and suppose $x \equiv y\left(\bmod \bigcap_{i=0}^{n}\left\{\Theta^{\mathbf{A}}\left(0, z_{i}\right)\right\}\right)$. Then for every $i \in\{0, \ldots, n\}$, we have $x \equiv y\left(\bmod \Theta^{\mathbf{A}}\left(0, z_{i}\right)\right)$, so by Lemma $2.6, x \backslash z_{i}=y \backslash z_{i}$ for all $i=0, \ldots, n$. Of course, $z_{j} \wedge z_{k}=0$ for all $0 \leq j<k \leq n$. By $\left(\mathrm{L}_{n}\right)$, $x=y$. Hence $\bigcap_{i=0}^{n}\left\{\Theta^{\mathbf{A}}\left(0, z_{i}\right)\right\}=\Delta_{A}$.

Conversely, suppose that for any family $\left\{z_{0}, \ldots, z_{n}\right\} \subseteq A$ of pairwise disjoint elements, $\bigcap_{i=0}^{n}\left\{\Theta^{\mathbf{A}}\left(0, z_{i}\right)\right\}=\Delta_{A}$. Assume $x, y \in A$ and $z_{0}, \ldots, z_{n} \in A$ are such that $x \backslash z_{i}=y \backslash z_{i}$ for all $i=0, \ldots, n$ and $z_{j} \wedge z_{k}=0$ for all $0 \leq j<k \leq n$. By Lemma 2.6, $x \equiv y\left(\bmod \Theta^{\mathbf{A}}\left(0, z_{i}\right)\right)$ for all $i=0, \ldots, n$. Hence,

$$
x \equiv y \quad\left(\bmod \bigcap_{i=0}^{n}\left\{\Theta^{\mathbf{A}}\left(0, z_{i}\right)\right\}\right) .
$$

As $\left\{z_{0}, \ldots, z_{n}\right\} \subseteq A$ is a family of pairwise disjoint elements, $\bigcap_{i=0}^{n}\left\{\Theta^{\mathbf{A}}\left(0, z_{i}\right)\right\}$ $=\Delta_{A}$, and so $x=y$. Thus $\mathbf{A} \models\left(\mathrm{L}_{n}\right)$.

Proposition 2.7 furnishes the equivalence $(3) \Leftrightarrow(4)$ in Theorem 1.1. This completes the proof of the main result of the paper.

Acknowledgment. The authors would like to thank Lukas Gerber for his careful proofreading of this paper.

\section{REFERENCES}

[1] Aglianò, P.: Fregean subtractive varieties with definable congruences. J. Austral. Math. Soc. Ser. A 71, 353-366 (2001)

[2] Balbes, R., Horn, A.: Stone lattices. Duke Math. J. 37, 537-545 (1970)

[3] Chajda, I., Halaš, R., Rosenberg, I.G.: Ideals and the binary discriminator in universal algebra. Algebra Universalis 42, 239-251 (1999)

[4] Grätzer, G.: Lattice Theory: First Concepts and Distributive Lattices. W. H. Freeman and Co., San Fransisco (1971)

[5] Lee, K. B.: Equational classes of distributive pseudocomplemented lattices. Canad. Math. J. 22, 881-891 (1970)

[6] Jones, G. T.: Pseudo Complemented Semi-Lattices. Ph.D. thesis, University of California at Los Angeles (1972)

[7] Lakser, H.: The structure of pseudocomplemented distributive lattices. I: Subdirect decomposition. Trans. Amer. Math. Soc. 156, 335-342 (1971) 
[8] Sankappanavar, H.P.: Remarks on subdirectly irreducible pseudocomplemented semilattices and distributive pseudocomplemented lattices. Math. Japon. 25, 519-521 (1980)

[9] Schmid, J.: Lee classes and sentences for pseudocomplemented semilattices. Algebra Universalis 25, 223-232 (1988)

[10] Schmid, J.: On amalgamation classes of pseudocomplemented semilattices. Algebra Universalis 29, 402-418 (1992)

\section{SPINKS}

Mathematical Institute, Sidlerstrasse 5, CH-3012 Bern, Switzerland e-mail: mspinksau@yahoo.com.au

\section{J. SCHMID}

Mathematical Institute, Sidlerstrasse 5, CH-3012 Bern, Switzerland e-mail: juerg.schmid@math.unibe.ch 